\title{
An Algorithm for Solving Torsional Vibration Problems Based on the Invariant Imbedding Method
}

\author{
Antonio Lopes Gama \\ Department of Mechanical Engineering, Universidade Federal Fluminense-UFF, 24210-000, Niterói, RJ, Brazil.
}

\author{
Rafael Soares de Oliveira \\ National Institute of Metrology-INMETRO, 25250-020, Duque de Caxias, RJ, Brazil.
}

\begin{abstract}
(Received 25 September 2012; provisionally accepted 20 August 2013; accepted 18 September 2013)
In this work the invariant imbedding method has been used to develop an algorithm to study the torsional vibration of non-uniform systems. The algorithm is based on the propagation, reflection, and transmission of waves in a stepped waveguide and is part of a procedure to transform two-point boundary value problems in initial value problems. Based on this approach, a continuous model has been developed and a simple, versatile, and robust algorithm has been constructed to solve torsional vibration problems of non-uniform shafts with circular crosssections. The proposed solution algorithm was extensively evaluated through comparisons with analytical solutions and the finite element method. The results show that the proposed method can provide the exact solution for uniform shafts with concentrated elements and accurate results for a wide variety of torsional vibration problems. Systems with continuously varying geometry may be approximated by stepped shafts. The proposed method can also be used to study the dynamic behaviour of others stepped systems.
\end{abstract}

\section{INTRODUCTION}

In the present paper, an approach usually applied to investigate the wave propagation in layered media is used to develop a continuous model to study the torsional vibration of nonuniform systems. ${ }^{1}$ The formulation of the proposed method considers that partial torsional waves propagate in opposite directions in a system with stepped changes in its properties as shown in Fig. 1. In the frequency domain, the governing equations are written in a state space form where the state variables are the angular displacement and twisting moment. The state matrix varies in a piece-wise constant fashion according to the properties of each segment of the rod. The part of the rod with continuously varying geometry (e.g., a conical part) is approximated by thin uniform segments. The solution of the state space equation is obtained by employing a discrete version of the Riccati transformation, which is a key ingredient in the invariant imbedding approach. ${ }^{2}$ This technique is used to transform two-point boundary value problems in initial value problems, and is also known as the method of sweeps, the Riccati method, or the factorization method. ${ }^{3,4}$ Based on this transformation, a recursive algorithm has been constructed for the solution, providing a simple and powerful computational method capable of solving problems of torsional vibration in circular non-uniform rods. ${ }^{5,6}$ Comparisons to analytical solutions and finite element results show that the proposed method can provide the exact solution for the torsional vibration of uniform shafts with concentrated elements and an approximated solution for shafts with a continuously varying geometry.

It must be pointed out that previous works have considered the propagation, reflection, and transmission of waves to solve vibration problems in finite inhomogeneous systems. ${ }^{7,8}$ It must also be mentioned that other methods to obtain the solu-

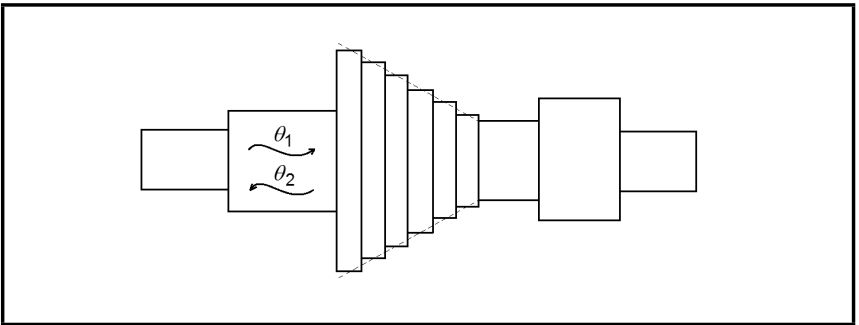

Figure 1. Stepped shaft with a conical part approximated by cylindrical segments.

tion for the torsional vibration of non-uniform rods have been presented in the literature. An analytical solution was provided by Pouyet and Lataillade ${ }^{9}$ for specific profiles of nonuniform rods, and the exact solution for more general cases has been obtained by Qiao et al. ${ }^{10}$ and Li. ${ }^{11}$ A continuous model for stepped shafts was proposed by Mioduchowski, ${ }^{12}$ and a general approach for stepped systems governed by the one-dimensional wave equation was presented by Bapat and Bhutani. ${ }^{13}$ A new exact approach for the analysis of torsional vibration of a non-uniform shaft carrying an arbitrary number of rigid disks has been proposed by Chen. ${ }^{14}$ Xiang et al. ${ }^{15}$ used the modified Riccati torsional transfer matrix method to calculate the torsional natural frequencies of a shaft system modelled as a chain consisting of an elastic spring with concentrated mass points.

As described in the foregoing sections, the main contribution of the present work is to provide a simple and concise algorithm that is able to solve a great variety of vibration problems. The algorithm can be easily implemented and used to solve the forced torsional vibration of non-uniform systems with classical or non-classical boundary conditions. Although the proposed method has been developed to study the vibra- 